\title{
Efecto de época del año (verano vs. invierno) en variables fisiológicas, producción de leche y capacidad antioxidante de vacas Holstein en una zona árida del noroeste de México
}

\author{
Effect of season (summer vs. winter) on physiological variables, \\ milk production and antioxidant capacity of Holstein cows in an arid zone \\ of northwestern Mexico
}

\author{
F Anzures-Olvera ${ }^{\mathrm{a}}$, U Macías-Cruz ${ }^{\mathrm{a}}$, FD Álvarez-Valenzuela ${ }^{\mathrm{a}}$, A Correa-Calderón ${ }^{\mathrm{a}}$, R Díaz-Molina ${ }^{\mathrm{b}}$, JA \\ Hernández-Rivera $^{c}{ }$, L Avendaño-Reyes $^{\mathbf{a}^{*}}$ \\ anstituto de Ciencias Agrícolas, Universidad Autónoma de Baja California, Baja California, México. \\ ${ }^{\text {b}}$ Facultad de Medicina, Universidad Autónoma de Baja California, Baja California, México. \\ ${ }^{\mathrm{c}}$ Facultad de Ciencias Naturales, Universidad Autónoma de Querétaro, Querétaro, México.
}

\begin{abstract}
SUMMARY
Heat stress (HS) induces the production of oxygen-derived free radicals associated with deleterious effects on the physiology, pathology and productivity of dairy cows. In order to evaluate physiological and productive responses of Holstein cows during summer and winter in an arid area, 40 lactating cows ( 100 to $150 \mathrm{~d}$ in milk) were randomly selected from a representative month per season of the year $(\mathrm{n}=20 / \mathrm{season})$. Data were analysed by ANOVA under a completely random experimental design, with season of the year as factor of interest (summer vs. winter), and a level of significance of 5\%. During winter time, cows were under their thermoneutral zone, whereas in summer they were under severe HS conditions. The skin temperatures of right side, buttock, udder and head, as well as raw milk were about $10^{\circ} \mathrm{C}$ higher in summer $(\mathrm{P}<0.05)$. Respiration rates were twice higher 106.87 \pm 0.05 vs. $47.74 \pm 0.06(\mathrm{P}<0.05)$ in summer when compared with winter, while the rectal temperature increased $1.6^{\circ} \mathrm{C}$ in summer. Cholesterol and TAS concentrations, ghecose, TOS and IEO were lower $(\mathrm{P}<0.05)$ in summer, while glucose TOS and IEO were higher $(\mathrm{P}<0.05)$ in summer than in winter. Milk production was reduced by more than half $(\mathrm{P}<0.05)$ in summer, and significant reductions of $42.6 \%$ in energy production in milk and $48.4 \%$ in the milk production adjusted to $3.5 \%$ fat were observed. The percentages of fat and protein in milk were higher $(\mathrm{P}<0.05)$ in summer. In conclusion, the effect of heat stress is evident in summer and has detrimental effects on physiological responses and productivity of lactating Holstein cows. Environmental innovations are needed to reduce the negative impact of high temperatures in this arid region of México.
\end{abstract}

Key words: heat stress, dairy cattle, antioxidant capacity.

\section{RESUMEN}

El estrés por calor (EC) induce la producción de radicales libres derivados del oxígeno que se asocian con efectos negativos en la fisiología, patología y productividad de vacas lecheras. Con el propósito de medir y comparar variables fisiológicas y productivas de vacas Holstein en verano e invierno en una zona árida, se seleccionaron aleatoriamente 40 vacas lactantes ( 100 a $150 \mathrm{~d}$ en leche) en un mes representativo de cada época del año ( $\mathrm{n}=20 /$ época). Los datos fueron analizados en un ANOVA bajo un diseño experimental completamente al azar, con época del año como factor de interés (verano vs. invierno), y un nivel de significación del 5\%. Las temperaturas de la piel en costado derecho, nalga, ubre y cabeza, así como de la leche, fueron $10{ }^{\circ} \mathrm{C}$ más altas $(\mathrm{P}<0,05)$ en verano. La tasa respiratoria fue dos veces mayor $(106,87 \pm 0,05$ vs. $47,74 \pm 0,06 ; \mathrm{P}<0,05)$ en verano, mientras que la temperatura rectal alcanzó $1,6^{\circ} \mathrm{C}$ más $(\mathrm{P}<0,05)$ en verano. Las concentraciones de colesterol y TAS fueron menores $(\mathrm{P}<0,05)$ en verano, mientras que la glucosa, TOS and IEO fueron mayores $(\mathrm{P}<0,05)$ en la misma época. La producción de leche se redujo en más del 50\% $(\mathrm{P}<0,05)$ en verano, observándose reducciones de 42,6\% en la producción de energía en leche y de 48,4\% en la producción de leche ajustada a 3,5\% de grasa. Se concluye que el efecto del EC es evidente y tiene un efecto perjudicial en la fisiología y productividad de vacas Holstein en producción, lo que hace necesario utilizar innovaciones ambientales para reducir este impacto negativo de las altas temperaturas.

Palabras clave: estrés calórico, ganado lechero, capacidad antioxidante, producción de leche.

\section{INTRODUCCIÓN}

En regiones áridas, la producción de leche se caracteriza por ser muy estacional debido a la variabilidad en las temperaturas ambientales durante el año (Avendaño-Reyes y col 2010). Generalmente la época de invierno en estas

Aceptado: 06.08.2014.

* Ejido Nuevo León, Valle de Mexicali, Baja California, México, C.P. 21705; 1ar62@hotmail.com zonas registra condiciones climáticas favorables para la productividad del ganado lechero, mientras que en verano las temperaturas se incrementan hasta valores que producen estrés por calor (EC). Así, en invierno la producción de leche se mantiene elevada y en verano se reduce drásticamente por efecto del EC. Como una medida para mantener la condición homeotérmica, el ganado lechero reduce su consumo de alimento y redistribuye parcialmente la energía disponible para activar mecanismos de termorregulación de tipos fisiológico, metabólico y endocrinológico, lo que 
le disminuye disponibilidad de energía para el proceso de galactogénesis y, consecuentemente, se presenta una baja en la producción de leche (Ganaie y col 2013). Una respuesta inmediata de la vacas lecheras a la presencia de EC es el incremento en la temperatura rectal, frecuencia respiratoria, y una reducción en el consumo de alimento, todo ello como medida para disipar la carga de calor y reducir la producción de calor metabólico.

Durante la última década se ha buscado explicar la reducción de producción de leche y la activación de mecanismos de termorregulación fisiológicos en verano como consecuencia del estrés oxidativo que el EC produce en el ganado lechero (Kumar y col 2011, Ganaie y col 2013). Se ha informado que el EC induce la producción de radicales libres derivados del oxígeno, estos se asocian a varios de los efectos negativos como son alta tasa respiratoria y temperatura corporal, bajo consumo de alimento, aumento de problemas metabólicos y una reducción en los parámetros productivos, reproductivos y resistencia a enfermedades (Bernabucci y col 2005, Castillo y col 2005). Como consecuencia, la intensificación en la producción de leche junto con factores ambientales adversos conlleva a un aumento en los niveles de estrés oxidativo en vacas lecheras (Kumar y col 2011).

Al respecto, Castillo y col (2006) informaron sobre alteraciones en la capacidad antioxidante de vacas lecheras durante la lactancia, demostrando que la concentración de malondialdehído como marcador de estrés oxidativo en plasma mantiene valores altos $(68,99 \pm 33,64 \mathrm{~lm} / \mathrm{L})$ durante la lactancia temprana, aunque con grandes variaciones interindividuales. Además, el aumento en la producción de radicales libres por efecto del EC puede conducir a un daño en el epitelio mamario, contribuyendo así a una disminución en la producción de leche (Aitken y col 2009). En otro estudio (Lakritz y col 2002), los niveles de glutatión oxidado en sangre se elevaron bajo condiciones de EC en bovinos. Asimismo, la actividad antioxidante total en plasma disminuyó cuando vacas lecheras fueron mantenidas en cámaras ambientales controladas a una temperatura de $29,5^{\circ} \mathrm{C}$ durante $7 \mathrm{~d}$. Se menciona que la información disponible acerca del estatus oxidativo de vacas Holstein en lactancia bajo condiciones de EC es escasa, existiendo solamente algunos reportes preliminares. Por tanto el objetivo de este estudio fue evaluar el efecto de época del año (verano vs. invierno) sobre algunas respuestas fisiológicas, la producción y composición de leche, así como la capacidad antioxidante de vacas Holstein bajo condiciones áridas del noroeste de México.

\section{MATERIAL Y MÉTODOS}

\section{LUGAR DE ESTUDIO}

El estudio se realizó en el establo comercial "Magaña", ubicado en el ejido Morelia, Valle de Mexicali, Baja California, México $\left(36^{\circ} 26^{\prime} \mathrm{N}, 115^{\circ} 11^{\prime} \mathrm{O}\right.$ y altitud de
$14 \mathrm{msnm})$. El clima en la región es árido y extremo, con temperatura máxima de $52^{\circ} \mathrm{C}$ en verano y mínima de $-6^{\circ} \mathrm{C}$ en invierno. La precipitación media anual es de $86 \mathrm{~mm}$, la que se considera escasa y se concentra principalmente en los meses de noviembre y diciembre (García 1985).

\section{ANIMALES, MANEJOY ALIMENTACIÓN}

Se utilizaron 40 vacas multíparas de raza Holstein, 20 en invierno y 20 en verano, estas tenían entre 2 y 3 partos y entre 100 y 150 d de producción de leche. Las vacas se alojaron en un corral $\left(5.082,48 \mathrm{~m}^{2}\right)$ abierto provisto de sombra, comederos en línea y bebederos. La sombra estaba ubicada al centro del corral $\left(691,78 \mathrm{~m}^{2}\right)$ y a lo largo del comedero $\left(564,72 \mathrm{~m}^{2}\right)$, el que abarcaba todo el lado norte del corral. Los animales fueron alimentados con $4 \mathrm{~kg}$ de concentrado comercial peletizado (93,6\% MS, $206 \mathrm{~g} / \mathrm{kg}$ MS PC y $1,98 \mathrm{EN}_{\mathrm{L}} \mathrm{Mcal} / \mathrm{kg} \mathrm{MS}$ ) ofrecido diariamente en dos porciones iguales utilizando un comedero grupal y heno de alfalfa (92\% MS, $154 \mathrm{~g} / \mathrm{kg}$ MS y 1,5 $\mathrm{EN}_{\mathrm{L}}$ Mcal/ kg MS) ad libitum.

El agua se ofreció sin restricción. El cuadro 1 muestra la relación de ingredientes de la dieta y su composición química.

\section{MEDICIONES Y MUESTREOS SANGUÍNEOS}

Los muestreos se realizaron tres veces en una semana de cada época, siendo durante verano el mes de julio y

Cuadro 1. Ingredientes y composición química de alimentos ofrecidos durante las dos épocas del año a los grupos de vacas Holstein.

Ingredients and chemical composition of the feeds offered during the two seasons of the year to the groups of Holstein cows.

\begin{tabular}{lc}
\hline Composición de la ración ofrecida (g/kg de alimento) & \\
\hline Heno de alfalfa & 600 \\
Concentrado comercial* & 400 \\
& \\
Composición química (g/kg) & \\
Materia seca (MS) & 981,6 \\
Proteína cruda (PC) & 180,0 \\
Grasa (extracto etéreo, EET) & 42,08 \\
Cenizas (CEN) & 72,6 \\
Fibra detergente neutro (FDN) & 339,1 \\
Energía neta de lactancia (EN ${ }_{\mathrm{L}}$ ) en Mcal/kg MS & 1,10 \\
Carbohidratos no estructurales (CNE**) & 366,2 \\
\hline
\end{tabular}

* Generaleche 958 (Agribrands Purina México, SA de CV, México, DF)

Contiene: $310 \mathrm{~g} / \mathrm{kg}$ de carbonato de calcio; $180 \mathrm{~g} / \mathrm{kg}$ de óxido de magnesio, $390 \mathrm{~g} / \mathrm{kg}$ fosfato dicálcico; $120 \mathrm{~g} / \mathrm{kg}$ de sal.

** Carbohidratos No estructurales $(\mathrm{g} / \mathrm{kg})=[1000-(\mathrm{CEN}+\mathrm{PC}+\mathrm{FND}$ $+\mathrm{EET})]$ 
durante invierno el mes de febrero, justamente cuando se presentan las más altas y las más bajas temperaturas ambientales durante el año. La información local acerca de las condiciones climáticas fue obtenida de la estación experimental climática del Instituto de Ciencias Agrícolas de la Universidad Autónoma de Baja California (ICAUABC), ubicada en el valle de Mexicali, a $6 \mathrm{~km}$ del sitio experimental. Las variables registradas fueron temperatura ambiental (TA) máxima y mínima por hora, y humedad relativa (HR) máxima y mínima. Con estas variables se calculó el índice de temperatura-humedad (ITH), siguiendo la fórmula propuesta por Hahn (1999) como:

$$
\begin{gathered}
\mathrm{ITH}=(0,81 \times \mathrm{TA})+\mathrm{HR} \\
(\mathrm{TA}-14,4)+46,4
\end{gathered}
$$

(Ecuación 1)

Las mediciones realizadas fueron condición corporal, variables fisiológicas (temperatura rectal, frecuencia respiratoria y temperatura de diferentes regiones de la piel) durante mañana y tarde (06:00 y 15:00 h) y producción y composición de la leche. La condición corporal se midió en una escala de 1 a 5 ( 1 = muy flaca y 5 = muy gorda; Wildman y col 1982), y siempre fue realizada por el mismo evaluador. La temperatura rectal se midió con un termómetro digital (Delta Track $\mathrm{CA}^{\mathrm{TM}}$, USA); la frecuencia respiratoria por conteo de movimiento en el espacio intercostal durante un minuto, y la temperatura de diferentes regiones de la piel (cabeza, ubre, flanco derecho y anca) con ayuda de una pistola de infrarrojo (Raytek, Modelo ST-20, CA ${ }^{\mathrm{TM}}$, USA) a una distancia de $1,5 \mathrm{~m}$ del animal. La producción de leche diaria (PL) también fue pesada colocando pesaleches (Waikato, Inter Ag, Hamilton, Nueva Zelanda) en la línea de ordeño durante la mañana y la tarde. La determinación de la composición de la leche se realizó mediante una muestra por vaca, la que se colectó en el ordeño de la mañana, determinando contenido de grasa $(\mathrm{G})$, proteína cruda (PC) y sólidos no grasos usando un analizador de leche (Lactichec ${ }^{\mathrm{TM}}$ LC-01, P\&P, USA). Además, en la misma muestra se midió temperatura de la leche con un termómetro digital (Delta Track, $\mathrm{CA}^{\mathrm{TM}}$, USA) y concentración de células somáticas con un equipo (DCC ${ }^{\mathrm{TM}}$, DeLAVAL, WC, USA). Con la información de producción y composición de la leche se calculó la producción de leche corregida a 3,5\% de grasa (LCG; Parekh, 1986) usando la fórmula:

$$
\begin{gathered}
\mathrm{LCG}=[((0,425 \times \mathrm{PL})+ \\
(16,425 \times((\% \mathrm{G} / 100) \times \mathrm{PL}))] \quad(\text { Ecuación } 2)
\end{gathered}
$$

También se calculó la energía en leche (EL; Tyrell and Reid, 1965) mediante la fórmula:

$$
\begin{gathered}
\mathrm{EL}(\mathrm{Mcal} / \mathrm{d})=(\{[(40,72 \times \% \mathrm{G})+ \\
(22,65 \times \% \text { PC })+102,77] / 1000\} \\
\text { x 2,204) x PL }
\end{gathered}
$$

(Ecuación 3)
Adicionalmente se colectaron muestras sanguíneas a partir de la vena coccígea en tubo vacutainer de $10 \mathrm{~mL}$ a las 16:00 h. Dichas muestras fueron transportadas en una hielera al laboratorio para ser centrifugadas a 3.500 rpm durante $15 \mathrm{~min}$ a temperatura de $10{ }^{\circ} \mathrm{C}$. El suero fue separado y colocado por duplicado en alícuotas de $2 \mathrm{~mL}$, para posteriormente almacenarlo a $-20{ }^{\circ} \mathrm{C}$ hasta la determinación de metabolitos (glucosa, colesterol y triglicéridos) y marcadores de estrés oxidativo (estado oxidativo total [TOS] y estado antioxidante total [TAS]). La concentración de metabolitos se determinó con un lector de química sanguínea (Modelo DT-60, Johnson \& Johnson Co., High Wycombe, UK). La concentración de TOS y TAS se determinó usando el método colorimétrico automatizado desarrollado por Erel (2005). Con la información de la concentración de TOS y TAS se calculó el índice de estrés oxidativo (IEO) usando la fórmula propuesta por Harma y col (2006), siendo:

$$
\mathrm{IEO}=\text { TOS/TAS } \quad(\text { Ecuación } 4)
$$

\section{ANÁLISIS ESTADÍSTICO}

Toda la información se sometió a un análisis de varianza bajo un diseño completamente al azar donde el tratamiento fue la época del año (invierno y verano), usando el procedimiento GLM del paquete estadístico SAS (SAS, 2004). Cuando se detectaron diferencias a un $\alpha=0,05$, se realizaron comparaciones de medias por medio de una prueba de " $\mathrm{t}$ " de Student. Cuando las probabilidades oscilaron entre 0,05 y 0,10 se consideró como tendencia.

\section{RESULTADOS}

En el cuadro 2 se resumen las principales variables climáticas para los períodos de estudio. La condición corporal no fue afectada por época $(\mathrm{P}<0,01)$, pero la temperatura rectal, la frecuencia respiratoria y las temperaturas de piel en las diferentes regiones del cuerpo fueron mayores $(\mathrm{P}<0,01)$ en verano (cuadro 3).

En invierno los promedios de producción de leche y de energía en leche fueron mayores $(\mathrm{P}<0,01)$, mientras que los promedios de temperatura en leche y del número de células somáticas, así como los porcentajes de grasa ( $\mathrm{P}=0,0003)$ y proteína cruda $(\mathrm{P}=0,033)$, fueron menores, comparado con el verano. Asimismo, el porcentaje de sólidos no grasos tendió $(\mathrm{P}=0,097)$ a incrementarse en el verano (cuadro 4).

La concentración de glucosa en suero fue mayor $(\mathrm{P}<0,01)$ en verano que en invierno, la de colesterol fue mayor $(\mathrm{P}<0,01)$ en invierno que en verano y la de triglicéridos no varió $(\mathrm{P}=0,1941)$ entre épocas. El TOS fue similar $(\mathrm{P}>0,05)$ entre las épocas, pero el TAS fue mayor $(\mathrm{P}<0,01)$ y el IEO menor $(\mathrm{P}<0,01)$ en invierno que en verano (cuadro 5). 
Cuadro 2. Promedio de temperatura ambiente máximas y mínimas, humedad relativa y unidades de ITH por mes del año. Average maximum and minimum ambient temperatures, relative humidity and THI units per month.

\begin{tabular}{|c|c|c|c|c|c|c|c|c|c|}
\hline \multirow{2}{*}{ Mes } & \multicolumn{3}{|c|}{ Temperatura $\left({ }^{\circ} \mathrm{C}\right)$} & \multicolumn{3}{|c|}{ Humedad relativa $(\%)$} & \multicolumn{3}{|c|}{ Índice temperatura-humedad } \\
\hline & Mínimo & Máximo & Promedio & Mínimo & Máximo & Promedio & Mínimo & Máximo & Promedio \\
\hline Enero & 8,2 & 22,2 & 14,8 & 19,2 & 55,0 & 36,2 & 52,7 & 66,4 & 58,7 \\
\hline Febrero & 8,4 & 21,1 & 14,4 & 19,9 & 56,0 & 36,1 & 52,9 & 65,3 & 58,3 \\
\hline Marzo & 13,8 & 27,7 & 27,7 & 16,3 & 51,2 & 32,5 & 57,7 & 72,8 & 72,8 \\
\hline Abril & 18,3 & 31,7 & 25,0 & 13,2 & 45,5 & 26,8 & 62,2 & 78,0 & 69,6 \\
\hline Mayo & 19,1 & 34,1 & 26,7 & 10,9 & 41,2 & 16,4 & 63,0 & 81,0 & 71,6 \\
\hline Junio & 24,5 & 40,7 & 32,8 & 8,7 & 39,0 & 21,2 & 69,0 & 90,4 & 79,3 \\
\hline Julio & 29,4 & 42,7 & 35,9 & 15,8 & 54,5 & 32,4 & 74,8 & 93,3 & 83,3 \\
\hline Agosto & 29,1 & 43,5 & 36,7 & 12,9 & 54,8 & 30,6 & 74,5 & 94,6 & 84,4 \\
\hline Septiembre & 27,5 & 40,9 & 34,4 & 16,8 & 56,5 & 34,5 & 72,5 & 90,6 & 81,4 \\
\hline Octubre & 20,9 & 35,0 & 28,0 & 15,2 & 67,6 & 37,5 & 59,8 & 82,0 & 69,7 \\
\hline Noviembre & 13,1 & 25,9 & 19,2 & 14,8 & 52,1 & 30,4 & 63,9 & 81,4 & 72,9 \\
\hline Diciembre & 7,6 & 20,4 & 14,6 & 36,2 & 84,9 & 62,6 & 59,8 & 82,0 & 69,7 \\
\hline
\end{tabular}

Cuadro 3. Efecto de época sobre respuestas fisiológicas de vacas Holstein lactantes.

Holstein cows.

Effect of season on physiological responses of lactating

\begin{tabular}{lrr}
\hline \multirow{2}{*}{ Variable } & \multicolumn{2}{c}{ Época } \\
\cline { 2 - 3 } & \multicolumn{1}{c}{ Invierno } & \multicolumn{1}{c}{ Verano } \\
\hline Condición corporal & $3,54 \pm 0,05^{\mathrm{a}}$ & $3,49 \pm 0,04^{\mathrm{a}}$ \\
Temperatura rectal $\left({ }^{\circ} \mathrm{C}\right)$ & $38,39 \pm 0,06^{\mathrm{a}}$ & $40,02 \pm 0,05^{\mathrm{b}}$ \\
Frecuencia respiratoria $(\mathrm{rpm})$ & $47,74 \pm 1,05^{\mathrm{a}}$ & $106,87 \pm 0,9^{\mathrm{b}}$ \\
Temperaturas de la piel & & \\
Nalga $\left({ }^{\circ} \mathrm{C}\right)$ & $27,14 \pm 0,32^{\mathrm{a}}$ & $36,38 \pm 0,27^{\mathrm{b}}$ \\
Flanco derecho $\left({ }^{\circ} \mathrm{C}\right)$ & $27,34 \pm 0,36^{\mathrm{a}}$ & $36,23 \pm 0,31^{\mathrm{b}}$ \\
Ubre $\left({ }^{\circ} \mathrm{C}\right)$ & $27,62 \pm 0,32^{\mathrm{a}}$ & $36,40 \pm 0,27^{\mathrm{b}}$ \\
Cabeza $\left({ }^{\circ} \mathrm{C}\right)$ & $26,74 \pm 0,24^{\mathrm{a}}$ & $36,25 \pm 0,21^{\mathrm{b}}$ \\
\hline
\end{tabular}

${ }^{\mathrm{ab}}$ Medias en hileras con diferente literal son diferentes $(\mathrm{P}<0,01)$.

Cuadro 4. Efecto de época sobre la producción y composición de la leche en vacas Holstein lactantes.

Effect of season on milk production and composition in lactating Holstein cows.

\begin{tabular}{lcc}
\hline \multirow{2}{*}{ Variable } & \multicolumn{2}{c}{ Época } \\
\cline { 2 - 3 } & \multicolumn{1}{c}{ Invierno } & \multicolumn{1}{c}{ Verano } \\
\hline Días en lactancia & $133,3 \pm 4.63$ & $137,6 \pm 6.32$ \\
Producción de leche $(\mathrm{kg} / \mathrm{d})$ & $34,07 \pm 1,01^{\mathrm{a}}$ & $15,96 \pm 0,87^{\mathrm{b}}$ \\
LCG 3,5\% $(\mathrm{kg} / \mathrm{d})$ & $31,47 \pm 1,30^{\mathrm{a}}$ & $18,07 \pm 1,12^{\mathrm{b}}$ \\
Energía en leche $(\mathrm{Mcal} / \mathrm{d})$ & $23,51 \pm 0,76^{\mathrm{a}}$ & $12,13 \pm 0,65^{\mathrm{b}}$ \\
Grasa $(\%)$ & $3,24 \pm 0,14^{\mathrm{a}}$ & $3,97 \pm 0,12^{\mathrm{b}}$ \\
Sólidos no grasos $(\%)$ & $9,23 \pm 0,08^{\mathrm{a}}$ & $9,41 \pm 0,07^{\mathrm{a}}$ \\
Proteína cruda $(\%)$ & $3,48 \pm 0,03^{\mathrm{a}}$ & $3,57 \pm 0,03^{\mathrm{b}}$ \\
Temperatura de leche $\left({ }^{\circ} \mathrm{C}\right)$ & $24,69 \pm 0,36^{\mathrm{a}}$ & $33,08 \pm 0,31^{\mathrm{b}}$ \\
Células somáticas & & \\
(Miles de células $/ \mathrm{mL})$ & $242,12 \pm 160,6^{\mathrm{a}}$ & $467,08 \pm 138,05^{\mathrm{b}}$ \\
\hline
\end{tabular}

ab Medias en hileras con diferente literal son diferentes $(\mathrm{P}<0,01)$. LCG a 3,5\% = Leche corregida a 3,5\% de grasa.
Cuadro 5. Efecto de época sobre concentración de metabolitos y marcadores oxidativos en suero de vacas Holstein lactantes.

Effect of season on serum metabolites and oxidative marker concentrations in lactating Holstein cows.

\begin{tabular}{lrc}
\hline \multirow{2}{*}{ Variable } & \multicolumn{2}{c}{ Época } \\
\cline { 2 - 3 } & \multicolumn{1}{c}{ Invierno } & \multicolumn{1}{c}{ Verano } \\
\hline Glucosa $(\mathrm{mg} / \mathrm{dL})$ & $51,70 \pm 0,95^{\text {a }}$ & $56,09 \pm 0,81^{\mathrm{b}}$ \\
Triglicéridos $(\mathrm{mg} / \mathrm{dL})$ & $49,53 \pm 4,69^{\text {a }}$ & $41,35 \pm 4,03^{\text {a }}$ \\
Colesterol $(\mathrm{mg} / \mathrm{dL})$ & $268,53 \pm 8,40^{\text {a }}$ & $186,00 \pm 7,22^{\mathrm{b}}$ \\
TOS $\left(\mu \mathrm{mol} \mathrm{H}_{2} \mathrm{O}_{2} \mathrm{Eq} / \mathrm{L}\right)$ & $4,62 \pm 0,28^{\text {a }}$ & $4,94 \pm 0,24^{\text {a }}$ \\
TAS $(\mu \mathrm{mol} \mathrm{Trolox} \mathrm{Eq/L)}$ & $685,35 \pm 23,2^{\text {a }}$ & $489,65 \pm 19,95^{\mathrm{b}}$ \\
IEO & $0,69 \pm 0,05^{\text {a }}$ & $1,02 \pm 0,05^{\mathrm{b}}$ \\
\hline
\end{tabular}

ab Medias en hileras con diferente literal son diferentes $(\mathrm{P}<0,01)$. TOS $=$ Estatus oxidante total, TAS $=$ Estatus antioxidante total, $\mathrm{IEO}=$ = Índice de estrés oxidativo.

\section{DISCUSIÓN}

Las condiciones ambientales extremas registradas tanto en invierno como en verano son típicas de regiones áridas, es decir, elevadas temperaturas combinadas con baja humedad durante el verano y viceversa en invierno. La mayor humedad relativa de invierno se debe a la distribución de lluvias que se registra en esta región del noroeste de México. En general, la información climática sugiere que mientras en invierno las vacas se encontraban dentro de su zona termoneutral y en condiciones óptimas para expresar su potencial de producción de leche, en verano se encontraron bajo EC severo, ya que la vaca lechera Holstein empieza a sufrir las consecuencias del calor cuando el ITH sobrepasa de 72 unidades (Bernabucci y col 2010). Estas condiciones de EC conllevan directamente a la activación de mecanismos termorregulatorios fisiológicos y metabólicos que favorecen una baja en la producción de leche, una mayor disipación de calor corporal y una reducción en la producción de calor metabólico (Ganaie y col 2013). 
De acuerdo con resultados previamente publicados (Leagates y col 1991, Tanaka y col 2007, Marai y col 2010, Ganaie y col 2013), las vacas monitoreadas en verano presentaron mayor temperatura rectal, frecuencia respiratoria y temperatura de las diferentes regiones de la piel donde se midió cuando se compara con las de invierno, esto último se explicaría por las condiciones ambientales registradas en verano $\left(14,4\right.$ vs. $\left.36,5^{\circ} \mathrm{C}\right)$. De hecho, la severidad el EC al que estuvieron sometidas las vacas durante el verano provocó que la tasa respiratoria de las vacas se incrementara en 1,5 veces. Se ha señalado que la tasa respiratoria bajo condiciones termoneutrales fluctúa entre 40 y 55 respiraciones/min para ganado lechero (Yousef 1985), mientras que en el presente estudio se registraron más de 100 respiraciones/min en verano. El aumento de la temperatura corporal y tasa respiratoria son mecanismos normales por los cuales las vacas disipan el calor para mantener su condición homeotérmica en ambientes cálidos, siendo $38,5^{\circ} \mathrm{C}$ la temperatura corporal considerada normal en bovinos (West 2003). En este estudio, ese valor no pudo ser mantenido por las vacas durante el verano, ya que su promedio alcanzó $40 \pm 0,05^{\circ} \mathrm{C}$. En vacas lecheras se encontró que la temperatura corporal se correlaciona positivamente con los cambios de temperatura durante las épocas del año (Johnson y col 1980).

Las condiciones de EC que prevalecieron en verano (junio, julio, agosto y septiembre con valores de ITH > 79 unidades) provocaron que la producción de leche y la energía en leche disminuyeran drásticamente comparado con el invierno. Esta es una respuesta biológica comúnmente observada en ganado lechero expuesto a altas temperaturas ambientales, ya que el incremento en su temperatura corporal interna, además de aumentar la tasa respiratoria para disipar calor corporal, también disminuye el consumo de alimento (Beede y Collier 1986) como medida para reducir la producción de calor metabólico, situación que se ha demostrado que afecta negativamente la síntesis de la leche (West 2003, Tanaka y col 2007, Avendaño-Reyes y col 2012). Una reducción de $25 \%$ en la producción de leche fue reportada cuando la temperatura ambiental fue superior a $28{ }^{\circ} \mathrm{C}$ (Kamiya y col 2005). En el presente estudio la temperatura ambiental promedio en verano fue de $36,7^{\circ} \mathrm{C}$ y la producción de leche disminuyó alrededor del $50 \%$, esto sugiere que, en conjunto con otros resultados, el nivel de producción de leche disminuye según las condiciones ambientales aumenten por encima de la zona de comodidad para ganado lechero (Kamiya y col 2005). Además se observó que los contenidos de grasa, proteína cruda y células somáticas en leche fueron mayores en verano que en invierno, ello muy posiblemente esté relacionado con el menor volumen de leche producido en verano. Una correlación negativa entre volumen secretado de leche y su composición química ha sido reportada previamente (Kamiya y col 2005). No obstante, la mayoría de los estudios consultados coinciden en mencionar que el porcentaje de grasa y proteína disminuye por efecto del EC (Bernabucci y col 2002 $2^{\mathrm{a}}$, Kadzere y col 2002, Joksimovié-Todorovic y col 2011). Esta discrepancia entre nuestros resultados y esos previamente publicados puede deberse a distintos factores como la cantidad y composición de concentrado ofrecido, calidad del forraje, tiempo de exposición al EC $\mathrm{y}$ temperatura ambiental. Las vacas en este estudio fueron alimentadas con una dieta (concentrado comercial y heno de alfalfa) de excelente calidad, de acuerdo con los resultados de su análisis bromatológico. En relación con el efecto de época sobre el número de células somáticas, también fue reportado un incremento en la concentración de células somáticas por efecto del EC de verano tanto en ganado Holstein como Jersey (Smith y col 2013). Similarmente, en otro estudio también reportaron dicho incremento en número de células somáticas bajo condiciones de altas temperaturas (Hammami y col 2013).

El mayor TOS en verano sugiere que el estrés calórico causó un aumento en la generación de especies reactivas al oxígeno, que normalmente son neutralizadas por los sistemas enzimáticos y no enzimáticos de los organismos. Se ha informado que el estrés oxidativo aumenta al final de la preñez y en la lactación temprana (Castillo y col 2005). Asimismo, el estrés oxidativo en las vacas lecheras aumenta en condiciones de temperaturas ambientales altas (Tanaka y col 2008). En un estudio realizado con vacas estresadas por calor (Bernabucci y col 2005), reportaron que la concentración de ácido ascórbico en plasma se redujo y la concentración de sustancias reactivas al ácido tiobarbitúrico aumentó al periparto ( -5 a $10 \mathrm{~d}$ después del parto), lo que indica un incremento en el estrés oxidativo en las vacas mantenidas en un ambiente de estrés calórico. Estos informes apoyan nuestra conclusión de que el estrés oxidativo aumenta en vacas después del parto. En este contexto, Bernabucci y col $\left(2002^{\mathrm{b}}\right)$ también han señalado que los marcadores de estrés oxidativo, las sustancias reactivas al ácido tiobarbitúrico, la actividad de la glutatión peroxidasa, los residuos sulfhidrilo y la actividad de la superóxido dismutasa en los eritrocitos aumenta en las vacas alrededor del parto durante la temporada estival.

El mayor TAS en invierno sugiere que las condiciones ambientales de esta época resultan en una menor generación de especies reactivas al oxígeno en ganado lechero en producción. Estos resultados produjeron un índice oxidativo total más alto en verano en relación con el invierno. Similarmente, Tanaka y col (2011) demostraron que el estrés oxidativo en vacas aumentó alrededor del parto en un ambiente caliente, especialmente durante el período de lactancia. Por su parte, Aitken y col (2009) encontraron que en vacas posparto, la expresión génica del prooxidante 15-lipoxigenasa 1 se incrementa en forma importante en el tejido mamario de vacas lecheras, además de observar altas correlaciones positivas entre distintas enzimas antioxidantes, lo que sugiere la generación de respuestas protectoras de estos antioxidantes a posibles estados inflamatorios. Estas respuestas pueden ser disminuidas cuando los partos de las vacas se presentan en 
la época cálida de verano. Similarmente, Sakatani y col (2012) observaron que en verano los niveles de glutatión, ácido tiobarbitúrico, superóxido dismutasa y glutatión peroxidasa en eritrocitos fueron menores en verano que en invierno en vacas no lactantes, concluyendo que las altas temperaturas del verano son un factor que determina un aumento en el estrés oxidativo en vacas Negras Japonesas.

Basado en estos resultados, se concluye que el EC en verano causa efectos negativos en la fisiología y productividad de las vacas lecheras Holstein. Esto indica la importancia de contar con estrategias ambientales para evitar la reducción en producción de leche durante los meses de altas temperaturas.

\section{REFERENCIAS}

Aitken SL, EL Karcher, P Rezamand, JC Gandy, MJ Vandehaar, AV Capuco, LM Sordillo. 2009. Evaluation of antioxidant and proinflammatory gene expression in bovine mammary tissue during the periparturient period. J Dairy Sci 92, 589-598.

Avendaño-Reyes L, FD Álvarez-Valenzuela, A Correa-Calderón, A Algándar-Sandoval, E Rodríguez-González, R Pérez-Velázquez, U Macías-Cruz, R Díaz-Molina, PH Robinson, JG Fadel. 2010. Comparison of three cooling management systems to reduce heat stress in lactating Holstein cows during hot and dry ambient conditions. Livest Sci 132, 48-52.

Avendaño-Reyes L, JA Hernández-Rivera, FD Álvarez-Valenzuela, U Macías-Cruz, R Díaz-Molina, A Correa-Calderón, PH Robinson, JG Fadel. 2012. Physiological and productive responses of multiparous lactating Holstein cows exposed to short-term cooling during severe summer conditions in an arid region of Mexico. Int J Biometeorol 56, 993-999.

Beede DK, RJ Collier. 1986. Potential nutritional strategies for intensively managed cattle during thermal stress. J Anim Sci 62, 543-554.

Bernabucci U, N Lacetera, B Ronchi, A Nardone. 2002 a Effects of the hot season on milk protein fractions in Holstein cows. Anim Res $51,25-33$.

Bernabucci U, B Ronchi, N Lacetera, A Nardone. 2002 ${ }^{\mathrm{b}}$. Markers of oxidative status in plasma and erythrocytes of transition dairy cows during hot season. J Dairy Sci 85, 2173-2179.

Bernabucci U, B Ronchi, N Lacetera, A Nardone. 2005. Influence of body condition score on relationships between metabolic status and oxidative stress in periparturient dairy cows. J Dairy Sci 88, 2017-2026.

Bernabucci U, N Lacetera, LH Baumgard, RP Rhoads, B Ronchi, A Nardone. 2010. Metabolic and hormonal acclimation to heat stress in domesticated ruminants. Anim Sci 4, 1167-1183.

Castillo C, J Hernández, A Bravo, M Alonso-López, V Pereira, JL Benedito. 2005. Oxidative status during late pregnancy and early lactation in dairy cows. Vet $J$ 169, 286-292.

Castillo C, J Hernández, I Valverde, V Pereira, J Sotillo, M AlonsoLópez, JL Benedito. 2006. Plasma malonaldehyde (MDA) and total antioxidant status (TAS) during lactation in dairy cows. Res Vet Sci 80, 133-139.

Erel O. 2005. A new automated colorimetric method for measuring total oxidant status. Clin Biochem 38, 1103-1111.

Ganaie AH, G Shanker, NA Bumla, RS Ghasura, NA Mir, SA Wani, GB Dudhatra. 2013. Biochemical and physiological changes during thermal stress in bovine. $J$ Vet Sci Technol 4, 2157-7579.

García E. 1985. Modificaciones al sistema de clasificación climática de Köeppen (para adaptarlo a las condiciones de la República Mexicana). $2^{\mathrm{a}}$ ed. Instituto de Geografía, Universidad Nacional Autónoma de México, DF, México.
Hahn GL. 1999. Dynamic responses of cattle to thermal heat loads. J Dairy Sci 82, 10-20.

Hammami H, J Bormann, N M'Hamdi, HH Montaldo, N Gengler. 2013. Evaluation of heat stress effects on production traits and somatic cell score of Holsteins in a temperate environment. J Dairy Sci 96, 1844-1855.

Harma MI, M Harma, O Erel. 2006. Are d-ROMs and FRAP tests suitable assays for detecting the oxidative status? Eur J Obstet Gynecol Reprod Biol 127, 271-275.

Johnson HD. 1980. Environmental management of cattle to minimize the stress of climate changes. Int J Biometeorol 24, 65-78.

Joksimović-Todorović M, V Davidović, S Hristov, B Stanković. 2011. Effect of heat stress on milk production in dairy cows. Biotech Anim Husbandry 27, 1017-1023.

Kadzere CT, MR Murphy, N Silanikove, E Maltz. 2002. Heat stress in lactating dairy cows: a review. Livest Prod Sci 77, 59-91.

Kamiya M, Y Iwama, M Tanaka, S Shioya. 2005. Effects of high ambient temperature and restricted feed intake on nitrogen utilization for milk production in lactating Holstein cows. J Anim Sci 76, 217-223.

Lakritz J, MJ Leonard, PA Eichen, GE Rottinghaus, GC Johnson, DE Spiers. 2002. Whole-blood concentrations of glutathione in cattle exposed to heat stress or's combination of heat stress and endophyteinfected tall fescue toxins in controlled environmental conditions. American J Vet Res 63, 799-803.

Leagates JE, BR Farthing, RB Casady, MS Barrada. 1991. Body temperature and respiratory rate of lactating dairy cattle under field and chamber conditions. J Dairy Sci 74, 2491-2500.

Marai IFM, AAM Haeeb. 2010. Buffalo's biological functions as affected by heat stress - A review. Livest Sci 127, 89-109.

Parekh HKB. 1986. A new formula for FCM (3.5\%) fat [Fat-corrected milk]. Indian J Anim Sci 56, 608-609.

Sakatani M, AZ Balboula, K Yamanaka, M Takashashi. 2012. Effect of summer heat environment on body temperature, estrous cycles and blood antioxidant levels in Japanese Black cow. Anim Sci J 83, 394-402.

SAS. 2004. SAS/STAT Guide for personal computers. Release 9.2. SAS Institute Inc., Cary, N.C., USA.

Smith DL, T Smith, BJ Rude, SH Ward. 2013. Short communication: Comparison of the effects of heat stress on milk and component yields and somatic cell score in Holstein and Jersey cows. J Dairy Sci 96, 3028-33.

Kumar BV, A Kumar, K Meena. 2011. Effect of heat stress in tropical livestock and different strategies for its amelioration. J Stress Phys Biochem 7, 45-54.

Tanaka M, Y Kamiya, M Kamiya, Y Nakai. 2007. Effect of high environmental temperatures on ascorbic acid, sulfhydryl residue and oxidized lipid concentrations in plasma of dairy cows. J Anim Sci 78, 301-306.

Tanaka M, Y Kamiya, T Suzuki, M Kamiya, Y Nakai. 2008. Relationship between milk production and plasma concentrations of oxidative stress markers during hot season in primiparous cows. J Anim Sci 79, 481-486.

Tanaka M, Y Kamiya, T Suzuki, Y Nakai. 2011. Changes in oxidative status in periparturient dairy cows in hot conditions. J Anim Sci $82,320-324$.

Tyrrell HF, JT Reid. 1965. Prediction of the energy values of cow's milk. J Dairy Sci 48, 1215-1223.

West JC. 2003. Effects of heat stress on production in dairy cattle. J Dairy Sci 86, 2131-2144.

Wildman EE, GM Jones, PE Wagner, RL Boman, HF Troutt, TN Lesch. 1982. A dairy cow body condition scoring system and its relationship to selected production characteristics. J Dairy Sci 65, 495-501.

Yousef MK. 1985. Stress Physiology in Livestock. In: Yousef MK (ed.) Basic Principles, Vol. 1. CRC Press. Boca Raton, FL, USA. 